\title{
Depression in the physically ill
}

(Index words: depression, antidepressants, continuing medical education, physically ill, management)

\section{Summary}

The risk of depression is increased in many medical and surgical conditions. Specific serotonin reuptake inhibitors are generally recommended as first line treatment in the physically ill. Care must be taken in prescribing antidepressants in the physically ill, because of sideeffects, hepatic and renal impairment, and potential for drug interactions.

\section{Introduction}

Major depression occurs in $5-10 \%$ of primary care patients and $10-14 \%$ of medical inpatients [1]. The risk of depression is increased in coronary artery disease, cerebrovascular accidents, neurological disorders, malignancies and diabetes. In the physically ill biological symptoms of depression - anorexia, weight loss, sleep disturbance and lethargy may be caused by the illness itself making the diagnosis of depression difficult. However, the diagnostic criteria are the same as for those without physical illness. Presence of other symptoms such as loss of interest, hopelessness, reduced self-esteem, guilt and suicidal ideas help in the diagnosis.

Tricyclic antidepressants (TCA) and selective serotonin reuptake inhibitors (SSRIs) are both effective in the treatment of depression in the physically ill. However, tricyclics, because of side-effects, are contraindicated in many conditions and SSRIs are generally recommended as first line treatment in the physically ill. There are little data regarding the use of newer antidepressants such as serotonin noradrenaline reuptake inhibitors (venlafaxine and duloxetine) and bupropion. Treatment may need to commence at lower doses due to increased sensitivity to side-effects in the elderly, in those with renal and hepatic dysfunction, and where there is risk of drug interactions.

\section{Cardiovascular disease}

Depression is a risk factor for both the development and the worsening of coronary heart disease. About $15-20 \%$ of patients with acute myocardial infarction experience a major depressive disorder. Depression increases all-cause mortality and cardiac mortality in those with ischaemic heart disease (IHD). Pathophysiological mechanisms responsible for this increase include increased platelet activation and aggregation, autonomic dysfunction causing elevated heart rate, low heart rate variability, exaggerated heart rate responses to physical stressors, high variability in ventricular repolarisation, and low baroreceptor sensitivity.
Tricyclic antidepressants and SSRIs are effective in the treatment of depression in patients with IHD. However, TCAs are known to affect heart rate variability, and cause orthostatic hypotension and conduction delays, and are best avoided in patients with IHD.

The SSRIs are relatively safe and effective in the treatment of depression in patients with IHD. There is evidence that antiplatelet effects of SSRIs may reduce the risk of ischaemic events. The SSRIs interfere with serotonin accumulation in platelets and normalises elevated indices of platelet activation and aggregation in patients with depression and IHD. Unlike the tricyclic antidepressants they do not cause orthostatic hypotension or arrhythmias. Antidepressants other than TCA and SSRIs have not been studied in detail in depressed patients with cardiovascular disease and their safety is unknown. Venlafaxine may cause hypertension and regular blood pressure monitoring is recommended.

\section{Stroke}

About one-third of patients develop depression after stroke. Peak prevalence is between 6 months and 2 years after stroke. Post-stroke depression (PSD) has significant effect on rehabilitation, motor recovery, activities of daily living, social life, and mortality [2].

Diverse mechanisms may be involved in the aetiology of PSD. One hypothesis postulates a deficiency of serotonin at central receptor sites. Cerebral lesions, aphasia, dependence in activities of daily living and social isolation too contribute to its development.

Diagnosis of PSD is made difficult by the unreliability of biological symptoms and the presence of cognitive and communicative defects caused by stroke. Use of special scales developed for use in stroke patients such as the structured assessment for depression in brain damaged individuals (SADBD) and the post-stroke depression rating scale (PSDRS) help in diagnosis. The Beck depression inventory (BDI) is useful for assessment of PSD because of its relatively low reliance on biological symptoms.

Establishment of a regular sleep pattern, a healthy diet, exercise and social support help in the treatment of depression. Both TCAs and SSRIs are effective in the treatment of PSD but SSRIs may be preferable because of the lower side-effect risk. Tricyclics cause orthostatic hypotension which can lead to falls, constipation and urinary retention which may be troublesome. TCAs are not recommended for use in patients with co-morbid IHD. 


\section{Involuntary emotional expression disorder (IEED)}

This manifests as sudden and short-lived episodes of crying, laughing or both which the patient cannot resist or prevent. It is a result of structural brain damage. IEED is common among patients with stroke and other neurological disorders, such as multiple sclerosis, amyotrophic lateral sclerosis, and traumatic brain injury. The recurrent crying spells may be mistaken for depression and doctors need to be able to differentiate between the two. There are no residual mood changes after an occurrence of crying or laughing.

\section{Renal disease}

Depression is the commonest psychiatric illness in patients with end-stage renal disease and chronic renal insufficiency. It is estimated that $5-22 \%$ of patients with end-stage renal disease have depression. Loss of role within the family and workplace, poor renal function, loss of mobility, physical skills and cognitive abilities, and impaired sexual function all contribute to the aetiology. Depression could alter the prognosis by influencing access to or utilisation of healthcare, modifying compliance with dialysis and medication, affecting nutritional status, and mediating changes in immune function [3].

In patients with renal disease tricyclic antidepressants are probably safer than SSRIs. Treatment should be started with a low dose and increased slowly. Of the SSRIs sertraline is not recommended in patients with renal impairment. In patients with a glomerular filtration rate (GFR) of $10-50 \mathrm{ml} / \mathrm{min}$ fluoxetine $20 \mathrm{mg}$ should be prescribed every other day. It should not be used in those with a GFR less than $10 \mathrm{ml} / \mathrm{min}$ [4].

\section{Hepatic disease}

Neurocognitive abnormalities and depression are frequently associated with chronic liver disease. The exact aetiology of depression in patients with liver diseases is unknown. All antidepressants are predominantly metabolised in the liver. As the risk of drug toxicity increases with the degree of hepatic impairment, lower starting and total doses of medication are recommended. Of the SSRIs low dose paroxetine seems to be the safest option. If fluoxetine is used it should be given every other day. Sertraline is extensively metabolised by the liver and is contraindicated in those with significant hepatic impairment. Most TCAs have a high first-pass clearance by the liver and lower starting doses are recommended [4].

\section{Diabetes mellitus}

Prevalence of depression in diabetes is estimated to be $9-27 \%$. Poor education, poor social support, financial stress and complications of diabetes increase the risk of depression. Depression may be an independent risk factor for developing type 2 diabetes.

Tricyclic antidepressants increase the appetite and induce weight gain. They are safe unless the diabetes is poorly controlled or is associated with significant cardiac disease [5]. In contrast SSRIs may cause appetite suppression and weight loss. Fluoxetine should be avoided, because of its potential for causing hypoglycaemia, particularly in type 2 diabetes, and its sideeffects of tremor, sweating and anxiety, which may be confused with hypoglycaemia [4]. Paroxetine and amitriptyline are effective in the treatment of diabetic neuropathy.

\section{Bleeding risk}

Because of the increased risk of bleeding SSRIs need to be used cautiously in patients on aspirin and NSAIDs. The increased bleeding risk is believed to be secondary to the blockade of serotonin reuptake in platelets and subsequent platelet dysfunction.

All SSRIs raise warfarin levels. Fluvoxamine has the most potent effect on warfarin but fluoxetine too has a potent effect. Raised INR has been reported within 10 days of starting fluoxetine. Sertraline and citalopram are the least likely to interact with warfarin [4].

\section{Drug interactions}

SSRIs are highly protein bound, and they and their metabolites inhibit the cytochrome P450 enzymes which are responsible for the metabolism of a range of drugs. Among the SSRIs paroxetine and fluoxetine have the highest potential and citalopram and sertraline the lowest potential for drug interactions involving hepatic metabolism [4]. Drug interactions should be carefully considered when combining drugs.

\section{Further reading}

1. Gelder MG, Cowen P, Harrison PJ. Shorter Oxford Textbook of Psychiatry. 5th ed. ed. Oxford: Oxford University Press; 2006.

2. Taylor D, Paton C, Kerwin R, South London and Maudsley NHS Trust, Oxleas NHS Foundation Trust. Prescribing guidelines. 9th ed. London

\section{References}

1. Katon W, Schulberg H. Epidemiology of depression in primary care. General Hospital Psychiatry 1992; 14: 237-47.

2. Turner-Stokes L, Hassan N. Depression after stroke: a review of the evidence base to inform the development of 
an integrated care pathway. Part 1: Diagnosis, frequency and impact. Clinical Rehabilitation 2002; 16: 231-47.

3. Kimmel PL. Depression in patients with chronic renal disease: what we know and what we need to know. Journal of Psychosomatic Research 2002; 53: 951-6.
4. Bazire S. Psychotropic drug directory: the professional's pocket handbook and aide memoire. 2005, Salisbury: Fivepin.

5. MacHale S. Managing depression in physical illness. Advances in Psychiatric Treatment 2002; 8: 297-306.

Raveen Hanwella and Varuni de Silva, Senior Lecturers, Department of Psychological Medicine, Faculty of Medicine, University of Colombo, Kynsey Road, Colombo 8.

Correspondence: RH, e-mail <raveenhanwella@yahoo.com.uk>. We declare that we have no conflicts of interest in regard to this article. 